Peterside $\mathrm{O}$

Kunle-Olowu OE

Adeyemi $\mathrm{OO}$

Akinbami FO

Omene J

\title{
Epidermolysis bullosa simplex: A case report
}

DOI:http://dx.doi.org/10.4314/njp.v39i4,9.

Accepted: 1st March 2012

Peterside O $\square$ )

Kunle-Olowu OE, Adeyemi OO, Akin-

bami FO, Omene J

Department of Paediatrics and Child

Health, Niger Delta

University Teaching Hospital,

Bayelsa State, Nigeria.

E-mail: docolyemen@yahoo.com.

Tel: +2348055855327

\begin{abstract}
Epidermolysis bullosa (EB) is a rare hereditary cutaneous disorder inherited mainly in an autosomal dominant fashion. ${ }^{1}$ It consists of a group of conditions that cause the skin to be fragile and blister easily. EB has been classified into three types namely; simplex, junctional and dystrophic ${ }^{2}$. Although all three types of EB have different causes, their symptoms are similar, manifesting as painful blisters and sores. Epidermolysis bullosa is a very rare condition but may probably be more common in clinical practice than reported in literature, especially in places like
\end{abstract}

Nigeria where there is under reporting of clinical cases. To the knowledge of the authors, there are few reported cases in Nigeria. 3, 4, 5 and none from Bayelsa State in the delta region of the country. We herein present a case of epidermolysis bullosa simplex (Dowling Meara type) in a 35 day old infant. This case is reported with the aim of increasing awareness of its existence in Nigeria and Bayelsa State in particular.

Key words: epidermolysis bullosa simplex, case, skin ulcers

\section{Case report}

N.L. was a 35 day old male, referred from Federal Medical Centre, Yenagoa with redness of the nail beds since birth, sores on the skin, and bleeding gums of a month duration.

He was born with long nails and redness of the nail beds which soon progressed to ulcers. The ulcers had been progressive since birth and his nails fell off after sometime. Sores on the skin started as blisters on the face which ruptured spontaneously to form ulcers. The ulcers increased in size and gradually progressed to involve the abdomen, umbilicus and perineum. Some of the ulcers healed without scarring while new ones formed. Parents noticed occasional gum bleeds which were aggravated by crying and resolved spontaneously.

He was admitted at the Federal Medical Centre (FMC), Yenagoa on the second day of life and was treated as a case of skin sepsis with intravenous antibiotics, topical antifungal and antibiotic creams without improvement. He was discharged home after nine days. When symptoms progressed, his parents took him back to FMC from where he was subsequently referred to the Niger Delta University Teaching Hospital (NDUTH).

Pregnancy was supervised at the Federal Medical Centre Yenagoa. Mother took only routine antenatal drugs and pregnancy was carried to term. Mother had prolonged rupture of membranes for about 48 hours before delivery by spontaneous vertex at a private hospital. He had been adequately immunized for age and was exclusively breastfed. He was the first and only child of unrelated parents in a monogamous family setting.

On examination, he was a well nourished active baby with necrotic ulceration of the nail beds of the fingers (fig. 1) and toes. The affected nails of both the hands and feet were completely destroyed. He also had ulcers on the gums with multiple scalds on the abdomen, umbilicus, trunk and perineum (fig. 2). All other systems were normal. An initial diagnosis of scalded skin syndrome was made and he was admitted and placed on intravenous antibiotics (cefuroxime and genticin) with syrup fluconazole. Skin ulcers were dressed with gentian violet lotion.

On review by the dermatologist, a diagnosis of epidermolysis bullosa simplex was made. His parents were counselled on care of the baby and were advised to clean the ulcers with normal saline and cover with mupirocin (bactroban) ointment. They were taught to wrap each finger loosely with Vaseline gauze to separate them. While on admission, some of the old ulcers healed without scarring but new ulcers started appearing. 
He was discharged after four days on oral antibiotic to come for follow up and skin biopsy the next week but was lost to follow up.

\section{Fig 1}

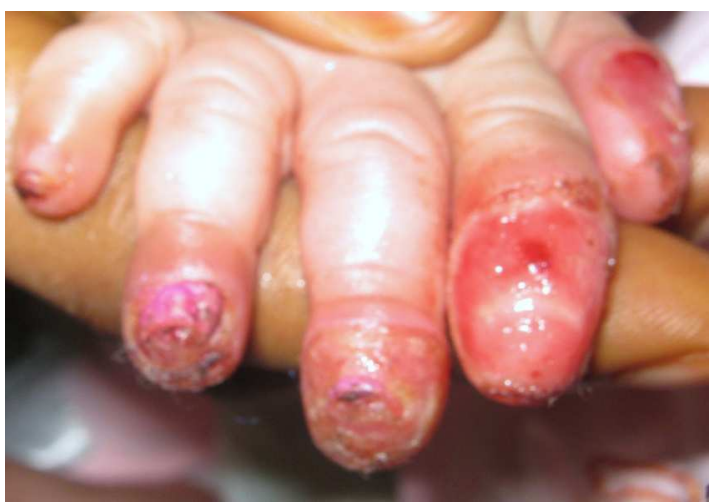

Fig 2

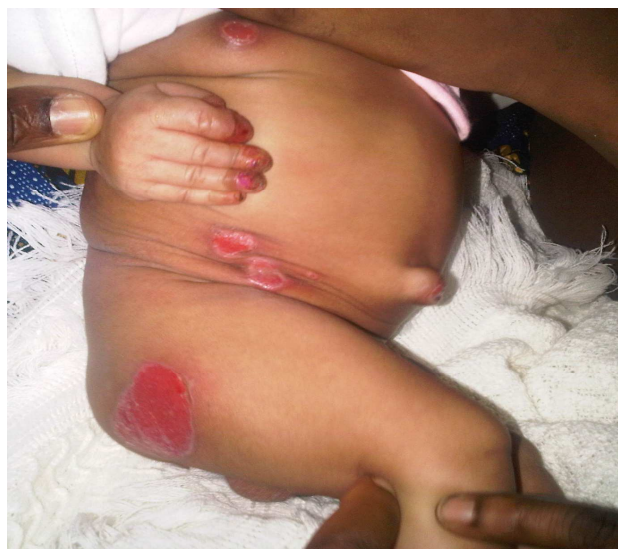

\section{Discussion}

Epidermolysis bullosa is a heterogenous group of mechanobullous disorders characterized by increased skin fragility and blistering of the skin and mucous membranes following minor or insignificant trauma or traction. It is caused by a mutation in the keratin or collagen gene. ${ }^{1}$

An estimated 50 in one million live births are diagnosed with epidermolysis bullosa. Of these cases, approximately $92 \%$ are epidermolysis bullosa simplex, $5 \%$ are dystrophic epidermolysis bullosa, $1 \%$ are junctional epidermolysis bullosa, and $2 \%$ are unclassified. Carrier frequency ranges from 1 in 333 for junctional, to 1 in 450 for dystrophic. Carrier frequency for simplex, is presumed to be much higher than in the other types. ${ }^{2}$

The simplex form of epidermolysis bullosa is inherited as an autosomal dominant trait. ${ }^{2}$ There are four types of epidermolysis bullosa simplex. The mildest form, known as the Weber-Cockayne type, is characterized by skin blistering that begins anytime between childhood and adulthood and is usually confined to the hands and feet. Later in life, skin on the palms and soles of the feet may thicken and harden (hyperkeratosis). In the Koebner type, blisters appear at birth or in early infancy and are more widespread. Another form of the disorder, called epidermolysis bullosa simplex with mottled pigmentation, is characterized by patches of darker skin on the trunk, arms, and legs that fade in adulthood. This form of the disorder also involves skin blistering from early infancy, hyperkeratosis of the palms and soles, and abnormal nail growth. The Dowling-Meara type is the most severe form of epidermolysis bullosa simplex. Extensive, severe blistering can occur anywhere on the body, including the inside of the mouth, and blisters may appear in clusters. Blistering is present from birth and tends to improve with age. Affected individuals also experience abnormal nail growth and hyperkeratosis of the palms and soles.

Symptoms of EB are first seen at birth as in our patient who presented with redness of the nail beds at birth. Our diagnosis was based on the history and physical examination. Our patient presented with the typical pattern of developing blisters on the hands and feet from birth with destruction of the nails. The blisters either occur spontaneously or in reaction to minor trauma. ${ }^{2}$ In our patient, the blisters on the skin were more on the trunk and perineum which may have been triggered by friction from his clothing and diapers while the sores on the nail beds occurred spontaneously. Gum bleeds were triggered by crying which would ordinarily not lead to bleeding in infants without this condition.

The variant of epidermolysis bullosa simplex (Dowling Meara type) found in our patient is the most severe form. ${ }^{2}$ In this form, extensive, severe blistering can occur anywhere on the body, including the inside of the mouth as in our patient. Blistering is present from birth and tends to improve with age. Affected individuals also experience abnormal nail growth and hyperkeratosis of the palms and soles. The outcome of the blisters in our patient may be difficult to ascertain as he was lost to follow up.

Oseni et $\mathrm{al}^{5}$ reported three similar cases in Owo. Their patients, like ours, were born with blistering and peeling of the skin from birth. As highlighted in their case report they also had similar diagnostic challenges. Similar to their experience, our patient was initially managed for skin sepsis and was discharged with no improvement. Diagnosis was subsequently made upon referral to a tertiary hospital but our patient was lost to follow up before the planned skin biopsy for histology was done.

Medenica et $\mathrm{al}^{6}$ in Serbia reported two cases of a 27 year old male Caucasian and a 24 year old female whom they had managed from birth. Like our patient, both patients presented with denuded skin on both feet with generalized blistering of the skin from birth. Both cases 
developed various problems ranging from pseudo fusion of the digits to difficulty with protrusion of the tongue. This highlights the problems our patient is likely to face in the future if he lives to the second decade.

In our patient, there was no family history of blistering disorders which points away from an autosomal dominant inheritance. There was also no history of consanguinity amongst the parents which may have been suggestive of an autosomal recessive inheritance. Kihiczak et $\mathrm{al}^{7}$ reported a case of an 18 month old female who presented with recurrent episodes of blistering, mostly of the hands and feet from birth. In their case, both parents were second cousins, suggesting an autosomal recessive inheritance. Unlike our patient, their patient seemed to have a milder variant of epidermolysis bullosa simplex as there was no history of oral bleeds. The condition was sporadic in our patient, probably due to new parental genetic mutations. Since he was the first child of his parents at the time he was seen, it was difficult to tell if other siblings from the same parents would develop the same manifestation. His parents were however offered genetic counselling which included the risk of recurrence in subsequent children.
Our case was different from that reported by Asish et al ${ }^{8}$ at Indira Ghandi Medical College. Their case was a 40 year old man who presented with symptoms from childhood unlike our patient who had symptoms from birth. Their patients lesions healed with scarring in contrast to ours where the lesions healed without scarring.

Treatment for epidermolysis bullosa simplex is mainly supportive. In our patient, his parents were counselled on avoidance of trauma and friction, wound management, infection control and nutritional support. A topical antibiotic cream, mupirocin (bactroban) was prescribed to prevent superimposed bacterial infection. Vaseline gauze was used to separate the fingers to prevent pseudofusion.

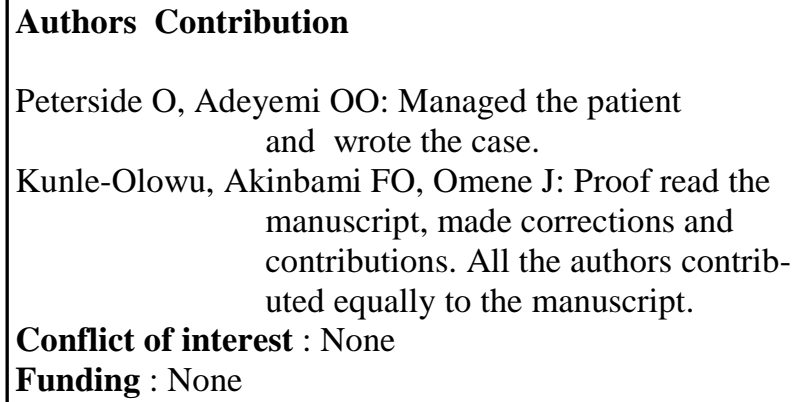

\section{References}

1. Bauer J, Schumann H, Sonnichsen K, Tomaske M, Bosk A, BrucknerTuderman L, Rassner G, Garbe C. Molecular diagnostics facilitate distinction between lethal and nonlethal subtypes of junctional epidermolysis bullosa: a case report and review of the literature. Eur $J$ Pediatr. 2002; 161: 672 - 679.

2. William J, Timothy B, Dirk E (2005). Andrews' Diseases of the Skin: Clinical Dermatology. (10th ed.). Saunders. ISBN: 0721629210

3. George AO. EB, three cases in an African family: medical, social and economic problems in management in a developing country. Trop Geogr Med. 1988; 40: 171 173
4. Kubeyinje EP, Onunu AN : Dystrophic epidermolysis bullosa with toenail deformity- report of a case in Benin City, Nigeria. Centr Afri J Med. 1991; 37: 156-158.

5. Oseni S. B, Oguntuase D. O, Onayemi O, Adelusola K. A, Omotoso O. A. Epidermolysis Bullosa In Owo, Nigeria: A report Of 3 cases. Int $J$ Pediatr and Neonatol. 2004; 4:1

6. Medenica L, Lens M: Recessive dystrophic epidermolysis bullosa: Presentation of two forms. Dermatology online Journal. 2008; 14: 3
7. Kihiczak N.I, Papadopoulos A.J, Schwartz R.A, Janniger C.K. Epidermolysis bullosa hereditary simplex: Case report. Dermatovenerologica. 2001; 10: 1 .

8. Ashish B, Vikran K.M, Nand L.S, Neha S, Anju L, Neelan G. Epidermolysis bullosa pruriginosa: Report of a rare case. Int J Dermatol. 2008; 6: 2 . 